\title{
Dasatinib as the salvage therapy for chronic myeloid leukemia with blast crisis and central nervous system involvement: A case report
}

\author{
SHIUE-WEI LAI, TZU-CHUAN HUANG, JIA-HONG CHEN, YI-YING WU and PING-YING CHANG \\ Department of Internal Medicine, Division of Hematology and Oncology, Tri-Service General Hospital, \\ National Defense Medical Center, Taipei, Taiwan, R.O.C.
}

Received April 24, 2014; Accepted January 22, 2015

DOI: $10.3892 / 01.2015 .2928$

\begin{abstract}
BCR-ABL tyrosine-kinase inhibitors are the first-line therapy for the majority of patients with chronic myelogenous leukemia (CML). Up to $20 \%$ of patients who have imatinib-treated CML in blast crisis (BC) experience a relapse in the central nervous system (CNS) due to the poor penetration of the drug by the blood-brain barrier. The present case reports a successful experience of using dasatinib-based combination therapy to treat a 22-year-old female who presented with initial symptoms of intermittent fever and easy bruising under the diagnosis of CML in BC. Although the patient eventually succumbed to profound sepsis, the CNS involvement was treated successfully using dasatinib-based combination therapy (cranial radiation and de-escalated intrathecal chemotherapy). This case demonstrates that dasatinib may be a viable option for those who are not medically fit for or are otherwise unwilling to receive high-dose chemotherapy. It appears that dose intensity is essential for optimal efficacy and should be maintained at $150 \mathrm{mg}$ daily as far as possible.
\end{abstract}

\section{Introduction}

Chronic myeloid leukemia (CML) is a myeloproliferative disorder that is associated with a unique translocation of chromosomes 9 and 22, resulting in the so-called Philadelphia chromosome $(\mathrm{Ph})$. This in turn results in the formation of a fusion protein product, BCR-ABL, which plays a key role in the pathogenesis of CML. Imatinib is the first-generation tyrosine kinase inhibitor (TKI) of BCR-ABL and is approved to treat $\mathrm{Ph}$-positive $\left(\mathrm{Ph}^{+}\right)$leukemia (1). Although the drug

Correspondence to: Dr Ping-Ying Chang, Department of Internal Medicine, Division of Hematology and Oncology, Tri-Service General Hospital, National Defense Medical Center, 325, Section 2, Cheng-Kung Road, Neihu 114, Taipei 100-116, Taiwan, R.O.C.

E-mail: p.ychang@yahoo.com.tw

Key words: dasatinib, chronic myeloid leukemia, blast crisis, central nervous system is highly active, it is noteworthy that $30-35 \%$ of patients do not have an optimal response due to treatment interruptions resulting from intolerance to the adverse effects (2).

Up to $20 \%$ of patients who have imatinib-treated CML in blast crisis $(\mathrm{BC})$ or $\mathrm{Ph}^{+}$acute leukemia experience a relapse in the central nervous system (CNS) $(3,4)$. This is a challenging issue in the imatinib era, and is due to the poor penetration of the drug by the blood-brain barrier (BBB) (4-10). Dasatinib and nilotinib, the second-generation TKIs, lead to a complete cytogenetic response (CyR) in $\sim 50 \%$ of imatinib-resistant patients and to major molecular remission in $20-30 \%(11,12)$. Furthermore, the two TKIs have demonstrated hematological and molecular responses higher than those of imatinib, and are approved for first-line treatment of newly diagnosed CML in the chronic phase (CP) $(13,14)$. At least one previous study has suggested that dasatinib crosses the BBB and is effective for $\mathrm{CNS} \mathrm{Ph}^{+}$leukemia (15). However, other studies have reported conflicting findings (16-21). The present study reports a successful experience of using dasatinib-based combination therapy to treat CNS involvement in a 22-year-old female with CML in BC. Written informed consent was obtained from the patient's family.

\section{Case report}

A 22-year-old female was diagnosed with CML in CP at Tri-Service General Hospital (Taipei, Taiwan) in November 2008 and was treated with imatinib $400 \mathrm{mg}$ daily. The patient showed poor compliance with frequent drug interruptions, and the best response during treatment was a major CyR. In October 2012, approximately four years after the initiation of therapy, the patient experienced a new onset of intermittent fever and easy bruising. Laboratory testing revealed marked leukocytosis, with a white blood cell (WBC) count of 330,000 $\mu \mathrm{l}$ (normal range, 4,500-11,000 $\mu \mathrm{l}$ ) and excessive peripheral myeloblasts (53.4\%; normal range, $0-1 \%$ ). A subsequent bone marrow (BM) biopsy revealed $60 \%$ myeloblasts (normal range, 0.3-5\%), and a diagnosis of $\mathrm{CML}$ in BC was made. No mutation was detected in the leukemic BCR-ABL transcripts. The patient received induction chemotherapy with seven days of $160 \mathrm{mg}$ cytarabine $\left(100 \mathrm{mg} / \mathrm{m}^{2}\right)$ and three days of $100 \mathrm{mg}$ daunorubicin $\left(60 \mathrm{mg} / \mathrm{m}^{2}\right)$. A repeat BM 

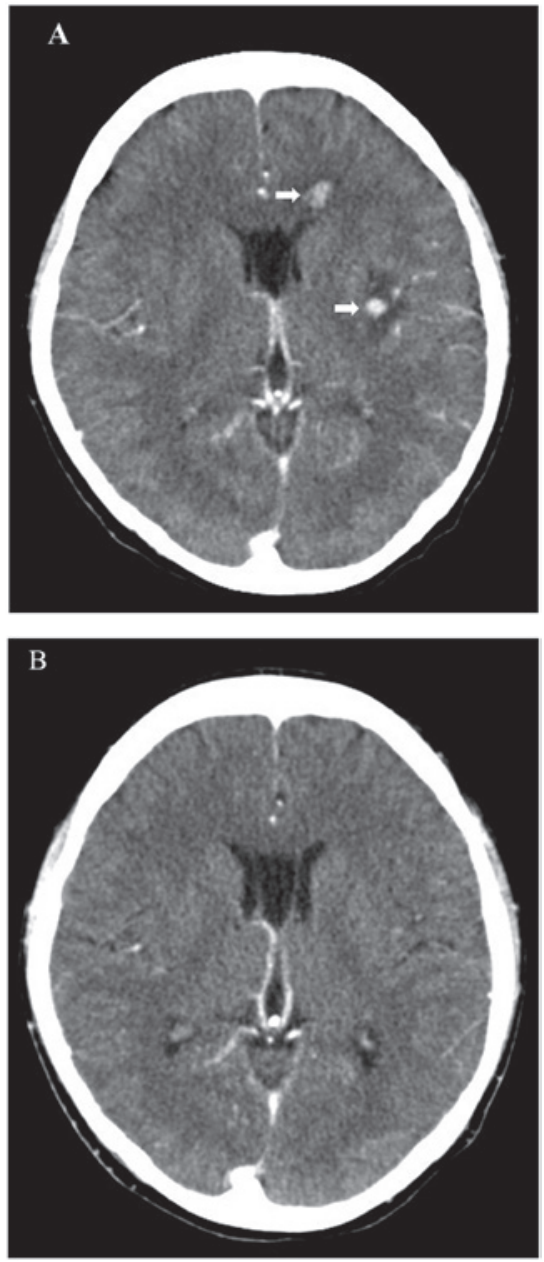

Figure 1. Computed tomography scan of the brain. (A) Multiple small enhancing nodules (white arrows), with mild perifocal edema in the bilatera cerebrum and left cerebellum. (B) Normal gray and white matter attenuation without evidence of abnormal enhancing lesions, demonstrated following dasatinib treatment and whole brain radiotherapy.

examination revealed partial remission after two weeks. Due to a new onset of left facial numbness, the patient underwent a computed tomography (CT) scan of the brain, which revealed multiple small enhancing nodules (Fig. 1A). Cerebrospinal fluid (CSF) examination revealed myeloperoxidase-positive blasts.

Due to a poor Eastern Cooperative Oncology Group performance status of 3, it was decided not to treat the patient with aggressive systemic or intrathecal therapy at that time. Instead, the patient was treated with $50 \mathrm{mg}$ dasatinib three times a day in combination with whole brain radiotherapy (total, 3,000 cGy). A repeat CT scan revealed near-complete resolution of the brain lesions (Fig. 1B). A repeat BM examination also revealed complete remission. During the treatment, only grade 1 hematological toxicities were noted. The patient was subsequently discharged and kept on the same dose of dasatinib.

One month later, the patient developed intermittent vomiting and a fever. Laboratory testing revealed pancytopenia, with a WBC count of 2,590 $\mu$ l (normal range, 4,500-11,000 $\mu \mathrm{l}$ ), a hemoglobin concentration of $8.4 \mathrm{~g} / \mathrm{dl}$ (normal range, 11.3-15.3 g/dl) and a platelet count of 109,000 $\mu \mathrm{l}$
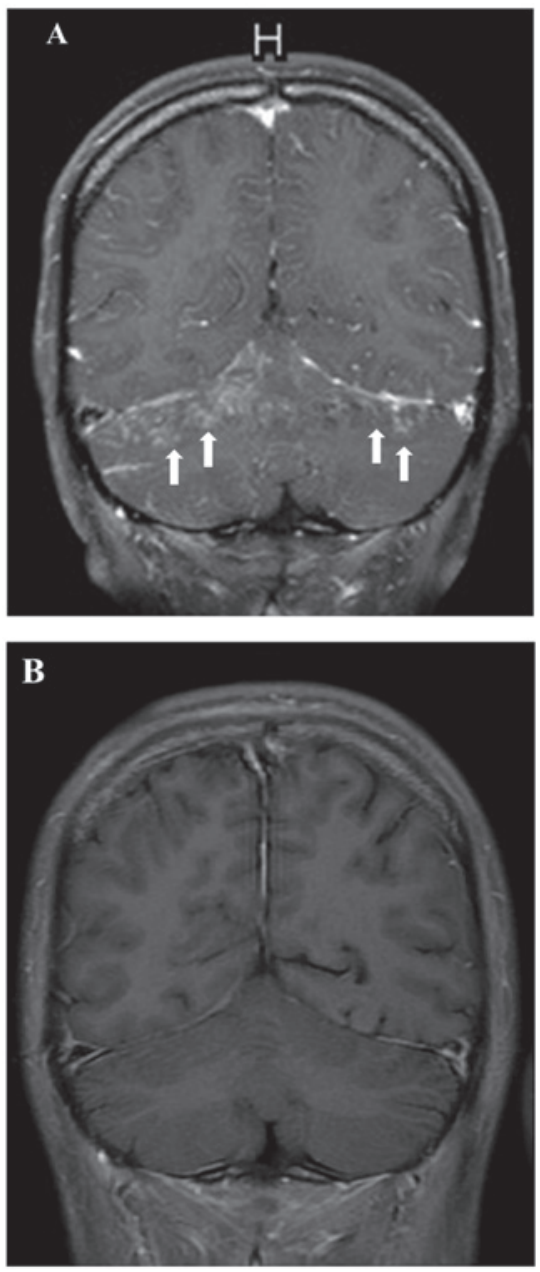

Figure 2. Gadolinium diethylenetriaminepentacetate-enhanced coronal T1-weighted image of the brain. (A) Diffuse leptomeningeal enhancement (white arrows) in the sulci of the bilateral cerebri and cerebelli. (B) Significant regression of the leptomeningeal enhancement, observed following treatment with dasatinib and intrathecal chemotherapy.

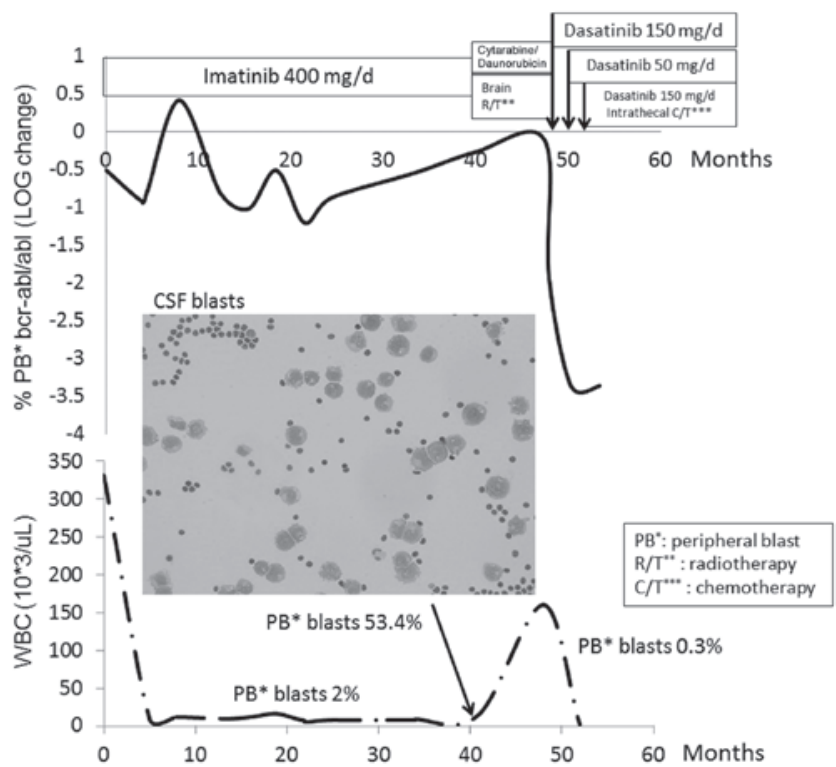

Figure 3. Clinical course of the present patient with Philadelphia chromosome-positive chronic myelogenous leukemia and recurrent central nervous system relapse while undergoing dasatinib combination therapy (CSF blast image; stain, Diff-Quik; magnification, x400). CSF, cerebrospinal fluid; WBC, white blood cell. 


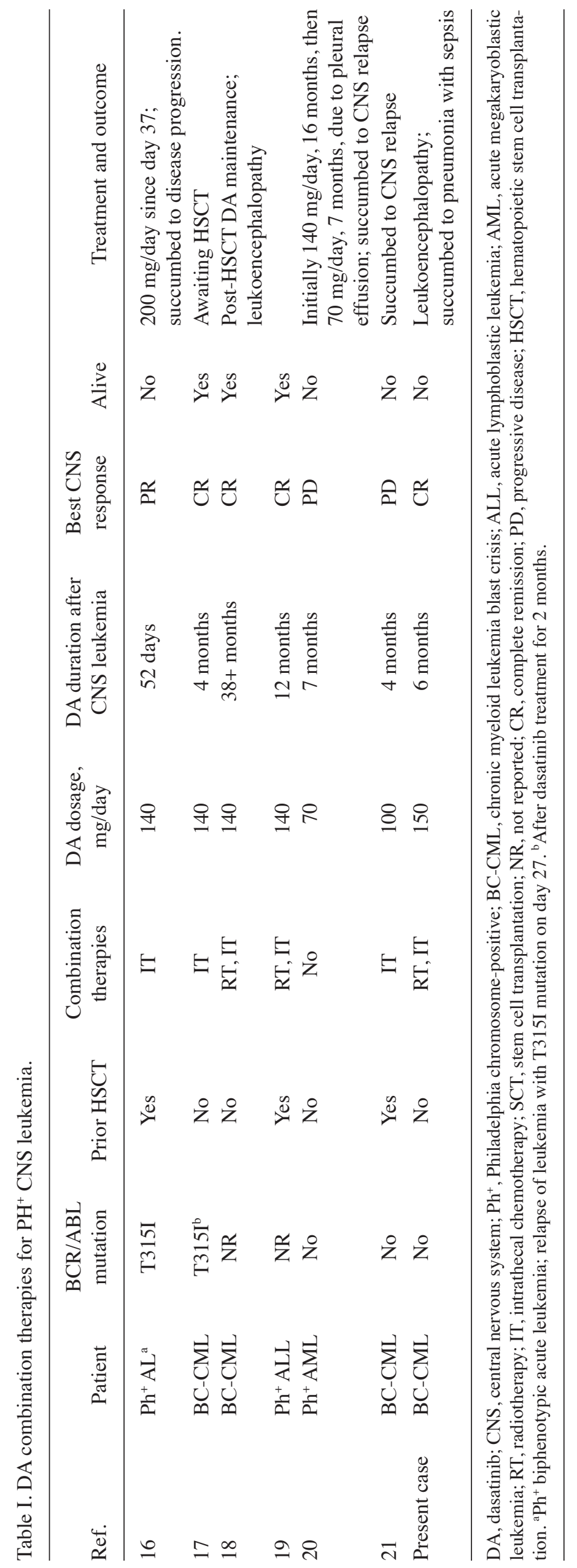


(normal range, 150,000-400,000 $\mu \mathrm{l}$ ). Since subsequent investigation did not reveal any notable cause, this was suspected to be due to the dasatinib treatment and so the dose was reduced. The next week, three days after the dose reduction of dasatinib, the patient experienced a generalized tonic-clonic seizure. Magnetic resonance imaging (MRI) of the brain revealed diffuse leptomeningeal enhancement (Fig. 2A). The patient became rapidly obtunded and required intubation for ventilator support. Following weekly intrathecal chemotherapy [methotrexate $(12 \mathrm{mg})$, cytarabine $(100 \mathrm{mg})$ and hydrocortisone $(50 \mathrm{mg})]$ for five courses, in combination with $50 \mathrm{mg}$ dasatinib three times daily, the patient's clinical condition gradually improved. Subsequently, the patient was successfully weaned off the ventilator and discharged. Furthermore, a repeat brain MRI revealed significant regression of the brain lesions (Fig. 2B). Grade 3 hematological toxicities were noted during the treatment. Two weeks after being discharged, the patient experienced confusion and generalized weakness. A brain MRI revealed diffuse leukoencephalopathy, but there was no evidence of CNS relapse on a repeat CSF examination. The patient was admitted to the hospital, but succumbed to pneumonia with profound sepsis two weeks after admission. The entire treatment course in correlation with BCR-ABL transcript levels is shown in Fig. 3.

\section{Discussion}

Acute leukemia with CNS involvement is not uncommon in clinical practice. Intrathecal chemotherapy, high-dose chemotherapy and radiotherapy are conventional treatments for CNS leukemia. However, a poor quality of life, significant systemic or CNS toxicity, a short response duration and ultimately mortality associated with refractory leukemia are common outcomes in a number of patients (22).

Imatinib is a substrate for the drug-eluting P-glycoprotein, which results in suboptimal penetration into the CNS, and the CSF levels of imatinib are 100-fold less than those achieved in plasma (6-8). This may explain why patients have CNS relapse in spite of achieving a complete response in the peripheral blood and BM. Dasatinib has greater potency (325-fold) than imatinib and can be therapeutically effective at a low or even subnanomolar concentration $(23,24)$. A study by Porkka et al demonstrated that dasatinib is associated with substantial clinical responses in patients with CNS leukemia and could significantly increase survival and control intracranial tumors in vivo (15). In addition to the present study, four separate case reports in Table I further support the potential benefit of dasatinib in $\mathrm{Ph}^{+} \mathrm{CNS}$ leukemia (16-19). In these four cases, the majority of patients received dasatinib combination therapies and all patients were administered $\geq 140 \mathrm{mg}$ dasatinib, daily (16-19). Nishimoto et al reported that dasatinib maintenance following allogeneic hematopoietic stem cell transplantation has the potential to prevent CNS relapse (18). In spite of these encouraging studies, it is sobering that several patients have progressive disease within months of starting therapy. Notably, Papageorgiou et al reported one case of $\mathrm{Ph}^{+}$acute megakaryoblastic leukemia who received $140 \mathrm{mg}$ dasatinib daily and maintained stable disease for 16 months, however, the patient experienced CNS relapse following treatment with a de-escalated daily dose of $70 \mathrm{mg}$ daily due to pleural effusion (20). Frigeri et al also presented a case of $\mathrm{Ph}^{+} \mathrm{CNS}$ leukemia in which dasatinib failed to prevent
CNS progression. However, this patient was administered $<100 \mathrm{mg}$ dasatinib daily during the treatment course (21).

While disease biology may play a significant role, it is vital to investigate whether other factors may be involved. One possibility may be the loss of CNS disease control with the lowering of the dasatinib dose. Indeed, it appears that among the cases reported in the literature, outcomes are poor when the dose is $<140 \mathrm{mg}$ a day (15-21). The most common reasons for decreasing the dose of dasatinib are adverse events, including cytopenia or pleural effusion (25). This was also observed in the patient in the present study, where progressive neurological deterioration occurred shortly after dasatinib dose reduction and a marked improvement was noted following re-escalation to $150 \mathrm{mg}$ once daily (Fig. 2). Although the overall experience with this issue is limited to the small number of cases in the literature, we believe that the available anecdotal data point to a requirement for a sufficient dose intensity of dasatinib for improved outcomes.

In conclusion, dasatinib may be a viable option for the management of patients with $\mathrm{Ph}^{+} \mathrm{CNS}$ leukemia, including those who are not medically fit for or are otherwise unwilling to receive high-dose chemotherapy. It appears that dose intensity is essential for optimal efficacy and should possibly be maintained at $150 \mathrm{mg}$ daily as far as possible. A well-designed, prospective study will aid in further clarifying this issue and better defining the role of dasatinib in this setting.

\section{Acknowledgements}

The authors would like to thank Dr Vivek R. Sharma, Division of Medical Oncology/Hematology, University of Louisville, School of Medicine (Louisville, KY, USA), for providing a critical review and comments on the manuscript.

\section{References}

1. Schiffer CA: BCR-ABl tyrosine kinase inhibitors for chronic myelogenous leukemia. N Engl J Med 357: 258-265, 2007.

2. Carella AM, Branford S, Deininger M, et al: What challenges remain in chronic myeloid leukemia research? Haematologica 98: 1168-1172, 2013.

3. Pfeifer H, Wassmann B, Hofmann WK, et al: Risk and prognosis of central nervous system leukemia in patients with Philadelphia chromosome-positive acute leukemias treated with imatinib mesylate. Clin Cancer Res 9: 4674-4681, 2003.

4. Leis JF, Stepan DE, Curtin PT, et al: Central nervous system failure in patients with chronic myelogenous leukemia lymphoid blast crisis and Philadelphia chromosome positive acute lymphoblastic leukemia treated with imatinib (STI-571). Leuk Lymphoma 45: 695-698, 2004.

5. Wolff NC, Richardson JA, Egorin M and Ilaria RL Jr: The CNS is a sanctuary for leukemic cells in mice receiving imatinib mesylate for Bcr/Abl-induced leukemia. Blood 101: 5010-5013, 2003.

6. Petzer AL, Gunsilius E, Hayes M, et al: Low concentrations of STI571 in the cerebrospinal fluid: a case report. Br J Haematol 117: 623-625, 2002.

7. Bornhauser M, Jenke A, Freiberg-Richter J, et al: CNS blast crisis of chronic myelogenous leukemia in a patient with a major cytogenetic response in bone marrow associated with low levels of imatinib mesylate and its N-desmethylated metabolite in cerebral spinal fluid. Ann Hematol 83: 401-402, 2004.

8. Dai H, Marbach P, Lemaire M, Hayes $M$ and Elmquist WF: Distribution of STI-571 to the brain is limited by p-glycoprotein-mediated efflux. J Pharmacol Exp Ther 304: 1085-1092, 2003.

9. Bujassoum S, Rifkind J and Lipton JH: Isolated central nervous system relapse in lymphoid blast crisis chronic myeloid leukemia and acute lymphoblastic leukemia in patients on imatinib therapy. Leuk Lymphoma 45: 401-403, 2004. 
10. Isobe $\mathrm{Y}$, Sugimoto K, Masuda A, Hamano Y and Oshimi K: Central nervous system is a sanctuary site for chronic myelogenous leukaemia treated with imatinib mesylate. Intern Med J 39: 408-411, 2009.

11. Hochhaus A, Kantarjian HM, Baccarani M, et al: Dasatinib induces notable hematologic and cytogenetic responses in chronic-phase chronic myeloid leukemia after failure of imatinib therapy. Blood 109: 2303-2309, 2007.

12. Kantarjian HM, Giles F, Gattermann N, et al: Nilotinib (formerly AMN107), a highly selective BCR-ABL tyrosine kinase inhibitor, is effective in patients with Philadelphia chromosome-positive chronic myelogenous leukemia in chronic phase following imatinib resistance and intolerance. Blood 110: 3540-3546, 2007.

13. Kantarjian H, Shah NP, Hochhaus A, et al: Dasatinib versus imatinib in newly diagnosed chronic-phase chronic myeloid leukemia. N Engl J Med 362: 2260-2270, 2010.

14. Saglio G, Kim DW, Issaragrisil S, et al: Nilotinib versus imatinib for newly diagnosed chronic myeloid leukemia. N Engl J Med 362: 2251-2259, 2010.

15. Porkka K, Koskenvesa P, Lundan T, et al: Dasatinib crosses the blood-brain barrier and is an efficient therapy for central nervous system Philadelphia chromosome-positive leukemia. Blood 112: 1005-1012, 2008.

16. Abdelhalim A, Barcos M, Block AW, et al: Remission of Philadelphia chromosome-positive central nervous system leukemia after dasatinib therapy. Leuk Lymphoma 48: 1053-1056, 2007.

17. Alimena G, Breccia M, Latagliata R, et al: Dasatinib in the management of lymphoid blast crisis of Philadelphia-positive chronic myeloid leukemia with multiple extra-medullary and intracranial localizations. Leuk Res 33: e134-e136, 2009.

18. Nishimoto M, Nakamae H, Koh KR, et al: Dasatinib maintenance therapy after allogeneic hematopoietic stem cell transplantation for an isolated central nervous system blast crisis in chronic myelogenous leukemia. Acta Haematol 130: 111-114, 2013.
19. Gutierrez-Aguirre H, Garcia-Rodriguez F, Cantu-Rodriguez O, Gonzalez-Llano O, Jaime-Perez J and Gomez-Almaguer D: Effectiveness of dasatinib in relapsed CNS, Ph+ ALL that is refractory to radiochemotherapy plus imatinib: a case report. Clin Adv Hematol Oncol 9: 875-878, 2011.

20. Papageorgiou SG, Pappa V, Economopoulou C, et al: Dasatinib induces long-term remission in imatinib-resistant Philadelphia chromosome-positive acute megakaryoblastic leukemia but fails to prevent development of central nervous system progression. Leuk Res 34: e254-e256, 2010.

21. Frigeri F, Arcamone M, Luciano L, Di Francia R, Pane F and Pinto A: Systemic dasatinib fails to prevent development of central nervous system progression in a patient with BCR-ABL unmutated Philadelphia chromosome-positive leukemia. Blood 113: 5028-5029, 2009.

22. Cortes J: Central nervous system involvement in adult acute lymphocytic leukemia. Hematol Oncol Clin North Am 15: 145-162, 2001.

23. O'Hare T, Walters DK, Stoffregen EP, et al: In vitro activity of Bcr-Abl inhibitors AMN107 and BMS-354825 against clinically relevant imatinib-resistant Abl kinase domain mutants. Cancer Res 65: 4500-4505, 2005.

24. Shah NP, Tran C, Lee FY, Chen P, Norris D and Sawyers CL: Overriding imatinib resistance with a novel ABL kinase inhibitor. Science 305: 399-401, 2004.

25. McCormack PL and Keam SJ: Dasatinib: a review of its use in the treatment of chronic myeloid leukaemia and Philadelphia chromosome-positive acute lymphoblastic leukaemia. Drugs 71 : 1771-1795, 2011 